\title{
Smart MEMS: Micro-Structures with Error-Suppression and Self-Calibration Control Capabilities
}

\author{
Andrei M. Shkel \\ Micro-Systems Laboratory \\ Department of Mechanical and Aerospace Engineering \\ Department of Electrical and Computer Engineering \\ University of California at Irvine \\ Irvine, California 92697
}

\begin{abstract}
Micro-sensors and actuators are differentiated from conventional sensors and actuators by their size, cost, and techniques used in their manufacture. The small size of MEMS is a convenience, a contribution to their potentially low cost, and a source of new conceptual challenges. Improvements in sensing structures, process technology, and circuit technology are presently addressed by the MEMS research community. Unavoidable, the next logical step in the MEMS evolution will be development of precision micro-systems by integrating a number of smart control functions including self-calibration, self-testing, structural compensation, and even communication with one or more other sensors located on the same chip. The control community will be the critical driving force in these developments. This paper gives an overview of functional blocks needed for Smart MEMS and provides several examples highlighting benefits of the paradigm.
\end{abstract}

\section{Introduction}

Micro-Electro-Mechanical Systems (MEMS) combining a large array of sensors and actuators controlled by sophisticated control electronics integrated on the same silicon chip are of increasing demand in everything from rigorously calibrated research and medical equipment to common household appliances. Yet, the current generation of MEMS is not reliable or fine-grained enough to meet applications performance needs.

Sensors and actuators play a significant role in our everyday lives as we gather information, process it and perform tasks. Yet, the successful application of a sensor is determined by its performance, cost and reliability. The cost of a sensor is often the single most important factor limiting its applicability. For example, at $\$ 20,000$, the cost of a conventional gyroscope has for many years precluded their use in automotive applications, consumer electronics, computer pe-

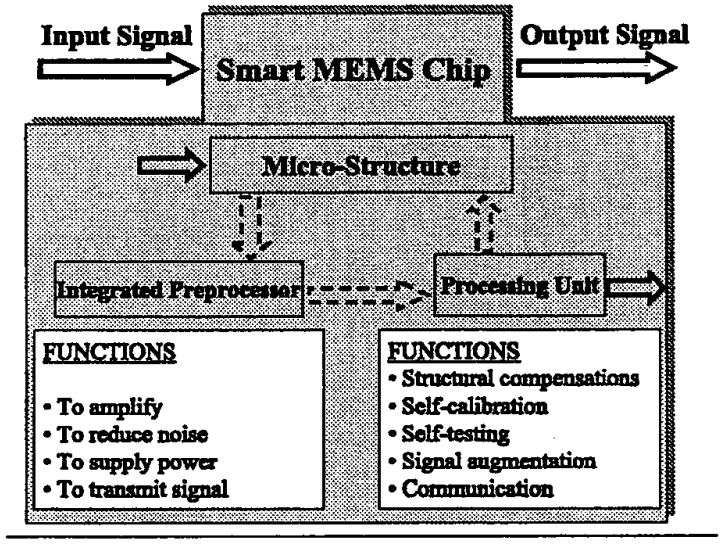

Figure 1: Architecture of a Smart MEMS Chip

ripherals, personal navigation systems, and robotics. Recent advances in silicon micromachining technology have raised the possibility of revolutionizing the field of inertial instruments by providing inexpensive, miniature gyroscopes to address market needs for low-cost medium performance inertial instruments.

The successful application of many types of solid-state sensors and actuators has stimulated the market but led to a demand for either a low unit cost or enhanced functionality to improve the market value. Both of these may be achieved through a higher level of device integration. In addition, many demanding applications required to solve more and more difficult sensing and actuation problems which require a higher level of processing power (i.e. more intelligence) than is achievable from current micro-systems. This paper gives an overview of functional blocks needed for implementation of the paradigm of Smart MEMS and provides several illustrative examples. 


\section{Functional Blocks of Smart MEMS}

What is a Smart MEMS? One can propose that a device onto which at least one sensing element and signal processing circuit has been integrated is a smart microsensor or smart micro-actuator (or, collectively, a smart MEMS). The problem with this definition is that most MEMS would be called smart, since some level of signal amplification and signal conditioning are needed in every micro-system. However, we would expect a smart MEMS to have integrated a number of functions including structural compensation, integration of signal processing, self-calibration, self-testing, communication with one or more other devices. The single goal to integrate these functions into a single device, or develop a smart MEMS, is to increase performance and reliability of the overall microsystem.

The basic arrangement of a Smart MEMS Chip is illustrated in Figure 1. Functional blocks and rational for implementing this functionality are discussed next.

Structural Compensation. Common processing techniques for fabricating MEMS includes bulk micromachining, wafer-to-wafer bonding, surface micromachining, and high-aspect ratio micromachining. Each of these fabrication processes involves multiple steps including the deposition, etching, and patterning of materials. As a rule, every fabrication step contributes to imperfections, which in most cases are reflected in the asymmetry and anisoelasticity of the mechanical structure. These asymmetries result in systematic perturbations in the form of parasitic mechanical and electrostatic forces. In order to improve performance, these structural imperfections need to be seamlessly detected, diagnosed, and compensated for. Ideally, an electronic block performing these functions will need to be integrated on the same silicon chip.

Integration of Signal Processing. Signal processing integrated onto a single chip can bring many advantages. The most compelling argument for the fabrication of an integrated system (e.g., a sensor) is to enhance the signal-to-noise ratio. The electrical power output from a microsystem is often low and susceptible to stray capacitance, inductance, and noise. In this case, the direct transmission of the output signal down a long interconnecting wire may be impractical. This is particularly true when, for example, a capacitive pick-up is used in a microsystem. The capacitance of a lateral resonant silicon structure may be only a few femtofarads. Thus, on-chip signal conditioning circuitry is highly desirable in order to remove the effect of the high input capacitance of transmission cables and subsequent instrumentation. Clearly, this will improve the response, sensitivity and resolution of the microsensor. There are other reasons for integrating the signal processing with the sensor, such as providing enhanced functionality at a lower cost, size, or weight.

Self-Testing and self-calibration. The ability of a sensor or actuator to test its functionality is highly desirable. Recent developments in the field of smart sensors are leading to sensors with some limited diagnostic capability. This is basically an ability of a sensor to determine whether it is functioning normally. A complete failure would usually be detected by the user as the output, either current or voltage, falls below its operating specification. However, in many cases a sensor can fail to perform adequately but provide a reasonable output. In these cases more sophisticated quality assurance is necessary. For instance, a noise characteristic (e.g., power spectral density) may be related to the physical property of the sensing element that is changing and effecting its performance and can thus be used to provide diagnostic information.

Multisensing and Signal Augmentation. Truly smart sensors and actuators should be able to improve their performance through the use of other sensors to monitor undesirable dependent variables. For example, the temperature-sensitivity of a microsensor could be ameliorated via the integration of a thermodiode near the sensing element.

A smart sensor can also eliminate spurious rather than systematic erroneous signals. For instance, an array of identical sensors can be employed and coupled to a microprocessor which calculates the average sensor output or perhaps discards any anomalous readings in a voting logic.

Communication. The ability of a sensor to communicate (i.e. exchange information with another device) is particularly useful. An intelligent sensor may be able to communicate with its operator and so provide valuable information about problems. Alternatively, an intelligent sensor or actuator may communicate with another device and so modify its own behavior. This type of intelligence can, in its simplest form, provide a warning of abnormal operating conditions, or more cleverly provide a feedback control mechanism. Intelligent sensors and actuators may provide a good level of control such as via a classical PID digital controller. An intelligent sensor may have some form of high level adaptive control strategy which permits the control parameters to be automatically updated with time. The implementation of a sensor which can warn its user, or adapt to environmental conditions, requires some decision making capability. In the simplest form of intelligence, sensors can use parametric data to make a decision. For example, a signal from a thermodiode can be used to provide an overload protection to a device which exceeds its normal operating temperature. However, the more intelligent sensors and actuators of the future may use non-parametric methods such as artificial neural net- 


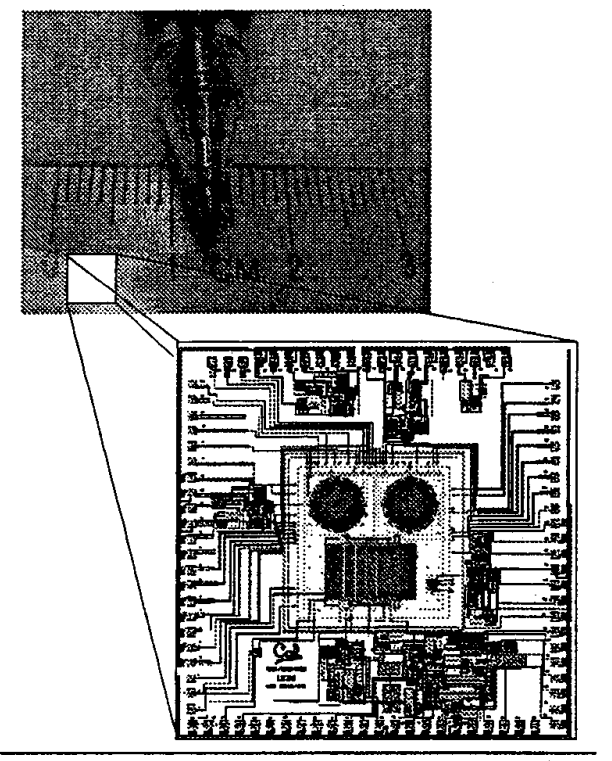

Figure 2: A prototype of fingernail sized "Smart Gyro Module" which includes three gyroscopes integrated with on-chip control electronics.

works [1] or a so-called expert systems [2].

\section{Smart Gyro Module}

Figure 2 illustrates a prototype of fingernail sized "Smart Gyro Module" which includes a MEMS gyroscope and several supporting sensors. All devices are integrated on the same silicon chip along with supporting control electronics. Next we describe the physics of micro-machined gyroscopes and discuss advanced control features that will lead to an improved performance and robustness of MEMS gyroscopes.

Gyroscopes are the most commonly used devices for measuring angular velocity and angular rotation in many navigation, homing, and stabilization applications [3].

In the basic mode of operation, the proof-mass, which constitutes the active portion of the sensor, is driven by an oscillator circuit at a precise amplitude and high frequency. When rotated, the proof-mass will be subjected to the Coriolis force:

$$
F=2 m \Omega \times V_{c}
$$

where $m$ - mass, $V_{c}$ - instantaneous radial velocity of the center of mass, $\Omega$ - input rate.

When an isotropic oscillator is allowed to freely oscillate in a rotating environment, the precession of the

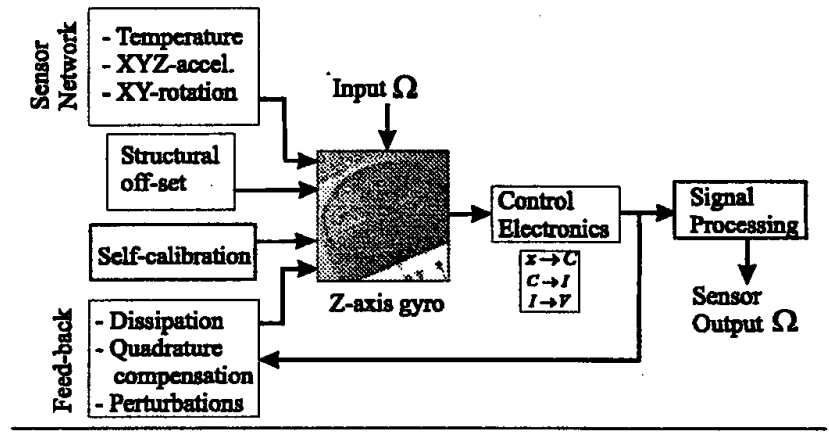

Figure 3: Design of sinart gyro chip supports self-test and self-calibration capabilities providing online off-set and gain control as well as online compensation of energy dissipation and quadrature compensation capabilities

line of oscillation provides a measure of the gyroscope's angle of rotation [4].

\subsection{Supported Functionality}

Functional blocks of a Smart gyro module is illustrated in Figure 3. Design of smart gyro chip supports selftest and self-calibration capabilities providing on-line off-set and gain control as well as on-line compensation of energy dissipation and quadrature compensation capabilities.

The gyroscope control system performs four basic feedback tasks: (1) initiates oscillations until the appropriate energy level is reached; (2) maintains the reached energy level; (3) compensates for quadrature deviation from the reference straight line of oscillations; (4) senses displacements and velocities in a pair of orthogonal directions. Several additional control functions need to be incorporated to increase robustness and performance of MEMS gyroscopes.

Ideally, the gyroscope oscillates along. a straight line and the Coriolis force causes the precession of this straight line, the precession is detected and information about the angle or angular rate is extracted [3, 4]. Non-idealities in the gyroscope, such as misalignment of the drive forces and anisoelasticity can cause ellipticity of the nominal straight line motion. Ellipticity of the gyroscope trajectory is undesirable because it directly enters into the measurements [5]. Thus, as a general rule, zero ellipticity is desirable. Angular momentum $\left[(\dot{x}, \dot{y})^{T} \times(x, y)^{T}\right]$ or ellipticity (quadrature) are good measures of deviation from a straight line oscillations:

$$
\begin{aligned}
P & =\frac{1}{2} \oint(x d y-y d x)=\frac{1}{2} \int_{0}^{2 \pi}(x \dot{y}-y \dot{x}) d t \\
& =\pi(x \dot{y}-y \dot{x})
\end{aligned}
$$

This measure of quadrature $P$ will be used for defin- 
ing a quadrature compensating controller. With some precautions discussed in the next section, the general goal of the quadrature control is to drive the area of the quadrature ellipsoid (1) to zero.

\subsection{General Control Strategy}

The gyroscope is sensitive to uncompensated defects which can arise due to the nature of the fabrication process, parasitic electrostatic forces and unwanted electrostatic cross coupling. These defects manifest themselves as unwanted terms in the spring and damping matrix. For example, symmetric off-diagonal terms in the stiffness matrix can cause the evolution of motion to be a precessing ellipsoid instead of a straight line, Figure 4(a). Therefore, the Coriolis force induced amplitudes along the $x$ - and $y$-axis no longer give us the correct information about the rotation rate.

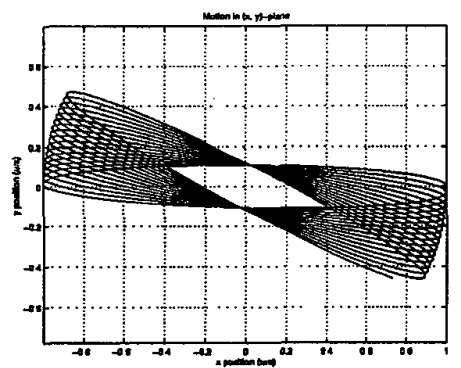

(a)

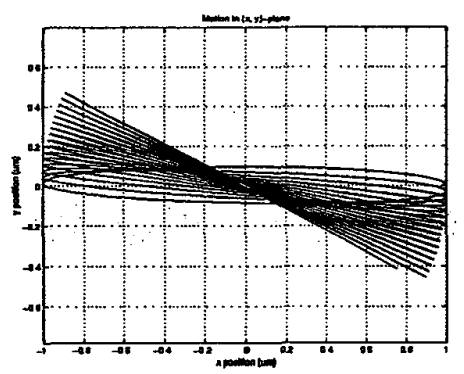

(b)

Figure 4: Performance of control minimizing quadrature. (a) The ellipsoid defining the quadrature precesses due to the Coriolis force; no control applied; (b) The area of quadrature ellipsoid converges to zero while the line of oscillation continue to precess; active feed-back control (2) is applied

At the same time this control should not interfere with the Coriolis force. Based on the result of classification of errors [5], the topology of the controller which will not interfere with the Coriolis force, while compensating for the quadrature, should have the form

$$
F_{\text {quadr }}=-\gamma_{1} \cdot P \cdot S^{T} \cdot q
$$

Here, $\gamma_{1}$ is a constant gain, $P$ is quadrature defined by
(1), $S$ is a skew-symmetric matrix, and $q$ is a displacement vector. This non-linear feedback control stabilizes the system to a manifold in the gyroscope phase space; the manifold corresponds to the straight line segment in $(x, y)$-plane. The performance of the quadrature compensation controller is illustrated in Fig. 4. In result of the quadrature control action, the area of the quadrature ellipsoid converges to zero, i.e. the ellipsoid approaches a straight line. Notice also that the quadrature control does not effect the rate of the precession.

In addition to such a control, one often uses an additional control to maintain constant overall energy of the system so that damping and other dissipative effects can be compensated. The deviation of the actual energy level of the system from the nominal can be defined by

$$
\Delta E=E_{0}-\frac{\omega_{n}^{2}\left(x^{2}+y^{2}\right)+\left(\dot{x}^{2}+\dot{y}^{2}\right)}{2}
$$

where $E_{o}$ denotes the nominal energy of the system normalized with respect to the effective mass. A control which will not interfere with the Coriolis force and will force the system to maintain the nominal energy level has the form:

$$
F_{\text {energy }}=-\gamma_{2} \cdot \Delta E \cdot \dot{q}
$$

This control force is proportional to the velocity and acts to cancel out the damping effects.

Due to structural sensitivity of the device to thermal gradients [6] and undesirable inertial perturbations, active sensing and compensation for these effects is necessary. In the gyro module, a sensor network will be integrated on the same chip. The network includes temperature, linear and angular acceleration sensors, and complementary rotational sensors. Readings from these sensors are continuously monitored and the gyroscope is compensated using a programmable reference voltages stored on-a-chip with a 3 bit resolution.

In summary, an advanced control architecture is needed for increasing robustness and performance of MEMS gyroscopes. The proposed control architecture, Figure 3 , is designed to compensate for manufacturing defects, electrostatic interferences, and external perturbations. The distinguishing feature of these controls is that they do not interfere with the measured Coriolis signal while performing assigned tasks.

\section{Tunable Micro-Interferometer}

Etalon, or Fabry-Perot Filter (FPF), is commonly used in telescopes and optical measurement devices. Tunable filters are also in high demand in telecommunication industry, where the goal is to filter wavelengths 
from $1500 \mathrm{~nm}$ to $1630 \mathrm{~nm}$, crucial for implementation of Dense Wavelength Division Multiplexing (DWDM). This device transmits a selected frequency of light by interference of multiple beams. It consists of two partially transmitting parallel mirrors separated by a medium, which forms a reflective cavity, Figure 5(a). A micro-machined prototype of the tunable interferometer is shown in Figure 5(b).

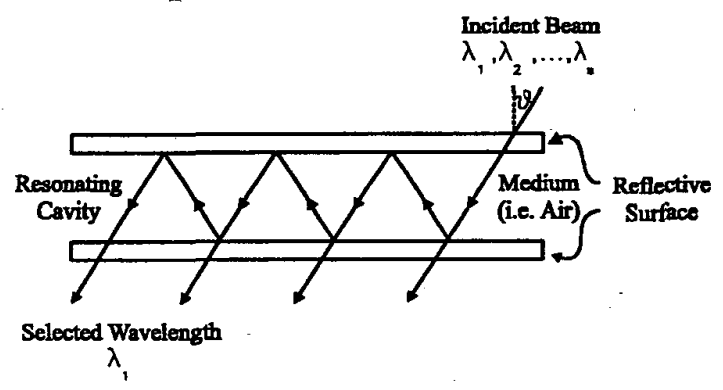

(a)

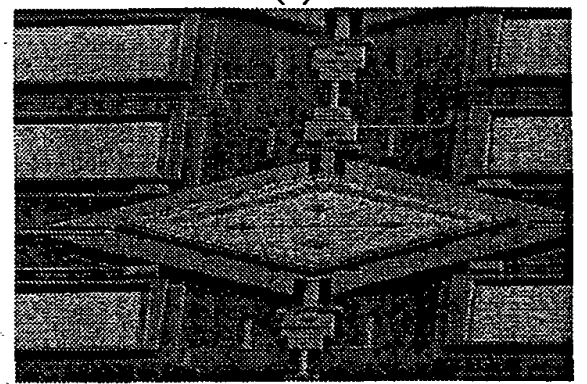

(b)

Figure 5: (a) Principle of operation of a Fabry-Perot interferometer; (b) A prototype of the tunable MEMS interferometer. Physical size of the device is less than 200 microns in diameter

Incident light enters the Fabry-Perot cavity, and undergoes multiple reflections. Each frequency, which makes up the incident light, goes through a constructive or a destructive interference. The constructive interference condition allows the frequency to be transmitted. Otherwise frequencies with destructive interference are filtered out.

Finesse determines the number of channels or fringes a FPF can transmit effectively. A higher finesse value results in higher resolution frequencies. Finesse depends on a variety of conditions, such as reflectivity, parallelism, and plate defects of mirrors. For the practical DWDM applications, the finesse should be in the range of 500-1000. That translates to very strict requirements: mirrors have to be extremely parallel all the time during the operation of the device with precision better than 0.1 micro-radians, step translation of the tunable etalon needs to be 1-2 [nm] over 1500-1620 [nm], the steps need to be controlled very accurately with very high precision $-0.25-0.3 \mathrm{~nm}$. In order to achieve such performance, error-suppression and selfcalibration control capabilities are essential and have to be embedded into the device. Figure 7 shows a block diagram of the signal processing that takes place in the capacitive tunable interferometer and its associated electronic circuitry. The interferometer is designed to have on-chip CMOS switched-capacitor circuitry to perform accurate capacitance-to-voltage conversion, signal conditioning and an automatic compensation for the device temperature using a reference voltage $V_{\text {ref }}$. The temperature-sensitivity of this smart interferometer $\alpha_{v}$ is related to the reference voltage $V_{\text {ref }}$ by,

$$
V_{\text {ref }}=V_{0}\left[1+\alpha_{v}\left(T-T_{0}\right)\right]
$$

and $\alpha_{v}$ is programmable with a 3 bit resolution.

Figure 6 illustrates the fringe broadening effect caused by temperature fluctuations in thermally uncompensated system [7]. If thermal compensation control is not used and reflectivity values of the reflective surfaces of interferometer are of $95 \%$ and $98 \%$, then $80 K$ increase in temperature $\left(B_{6}\right)$ decreases effective finesse by approximately $16 \%$ and $47 \%$, respectively.

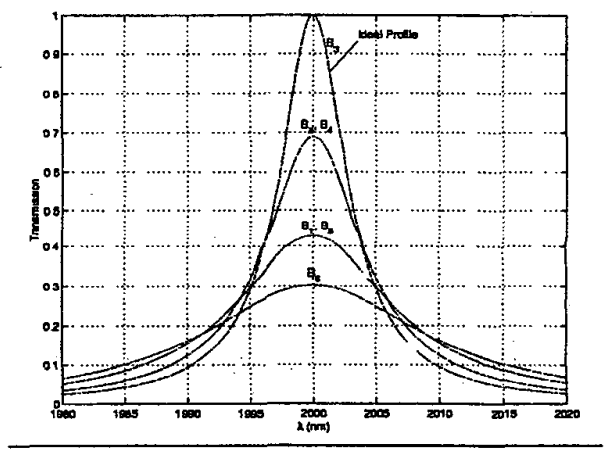

Figure 6: Fringe broadening effect as a result of thermal expansion.

This example illustrates that active thermal control is absolutely required for this device. Using advantages of micro-machining technology, a thermal sensor can be integrated on the same chip with the interferometer and used to compensate for undesirable effects using the approach discussed below. The control architecture of the tunable Febry-Perot filter is illustrated in Figure 7.

The purpose of the control system is to keep two reflective mirrors perfectly parallel to each other. Optical feed-back architecture can be used to solve this task. The photo-detector uses a predefined reference frequency signal. The sequence of equally spaced transmission picks ( $100 \mathrm{GHz}$ apart in our case) can be implemented using, e.g., ITU Grid. When ITU Grid 


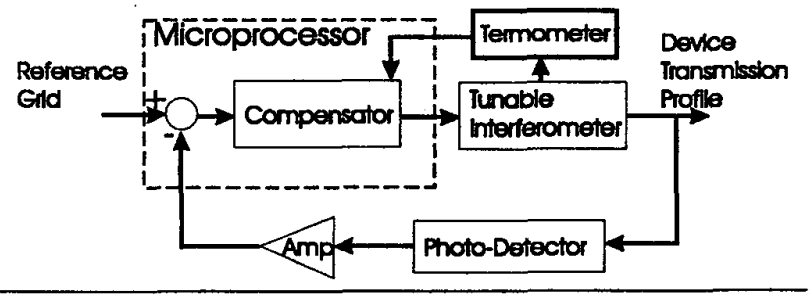

Figure 7: Schematic of a tunable Febry-Perot Interferometer

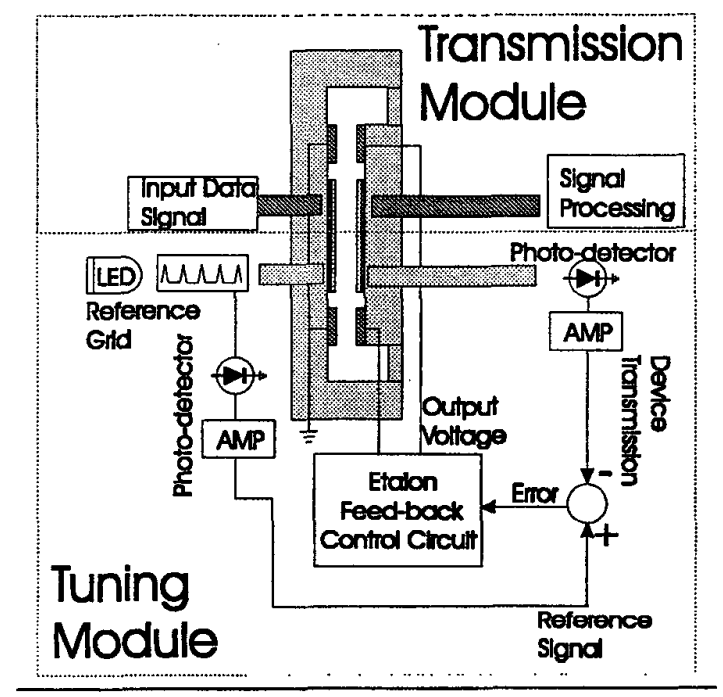

Figure 8: Block-diagram for feed-back controlled tunable Febry-Perot Interferometer

is lighted with LED, a sequence of modulated light wave is formed (called a reference grid). Two photodetectors are used in this configuration. The first is used to convert the reference light wave grid to reference current (or voltage). The second photo-detector measures intensity of the light wave resonating in the etalon cavity. The feed-back control architecture is based on maximization of the transmitted intensity of light given by the Airy function

$$
T=\left(1-\frac{A}{(1-R)}\right)^{2} \frac{1}{1+\frac{4 R \sin ^{2}\left(k d \cos \left(\theta-\frac{\pi}{2}\right)\right)}{(1-R)^{2}}}
$$

where $A$ is mirror absorption, $T$ is mirror transmittance, $R$ is mirror reflectivity, and $k$ equals to $2 \pi / \lambda$. From examination of Eq. (6), intensity is influenced by cavity gap, incident beam angle, and the refractive index. By taking advantage of these parameters, high precision sensing or light modulating applications can be realized.

\section{Conclusion}

Integrated sensors offer the prospect of considerable leverage on the larger microelectronic industry and are an important key in extending it into new areas. Improvements in sensing structures, process technology, circuit technology, and system organization in this evolution will all be important. Implementation of a smart control architecture will become necessary for improving performance of the next generation micro-sensors and actuators.

\section{Acknowledgements}

The author would like to thank Roger T. Howe and Roberto Horowitz, both from the University of California at Berkeley, for useful discussions on design and control of MEMS gyroscopes. The author would also like to acknowledge contribution of Chris Painter and Jung-sik Moon, from the University of California at Irvine, for their modeling efforts.

\section{References}

[1] T. Khanna. "Foundations of Neural Networks". Addison-Wesley Publishing Company Inc., USA, 1990.

[2] R. F. Forsyth. "Expert Systems: Principles and Case Studies". Chapman and Hall, London, 1984.

[3] A. Shkel. Micromachined gyroscopes: Challenges, design solutions, and opportunities. 2001 SPIE Annual International Symposium on Smart Structures and Materials, 2001. (Invited Paper) March, 2001, Newport Beach, CA.

[4] A. Shkel and R. T. Howe. Micromachined gyroscope for angle measurements. Patent Pending Application, June 1998. UC-Berkeley Office of Technology and Licensing. Case Number 98-046-3.

[5] A. Shkel, R. T. Howe, and R. Horowitz. Modeling and simulation of micromachined gyroscopes in the presence of imperfections. Intern. Conf. On Modeling and Simulation of Microsystems (MSM'99), April 1999. Puetro Rico, U.S.A.

[6] C. Painter and A. Shkel. Structural and thermal analysis of a mems angular gyroscope. 2001 SPIE Annual International Symposium on Smart Structures and Materials, 2001. March, 2001, Newport Beach, CA.

[7] J. Moon and A. Shkel. Analysis of imperfections in a micromachined tunable-cavity interferometer. 2001 SPIE Annual International Symposium on Smart Structures and Materials, 2001. March, 2001, Newport Beach, CA. 